\title{
Black hole scalarization from the breakdown of scale invariance
}

\author{
Carlos A. R. Herdeiro ${ }^{1}$ and Eugen Radu ${ }^{2}$ \\ ${ }^{1}$ Centro de Astrofísica e Gravitação-CENTRA, Departamento de Física, Instituto Superior Técnico-IST, \\ Universidade de Lisboa-UL, Avenida Rovisco Pais 1, 1049-001 Lisboa, Portugal \\ ${ }^{2}$ Center for Research and Development in Mathematics and Applications (CIDMA), \\ Department of Mathematics, Campus de Santiago, 3810-183 Aveiro, Portugal
}

(Received 16 January 2019; published 19 April 2019)

\begin{abstract}
Electrovacuum black holes are scale invariant; their energy-momentum tensor is traceless. Quantum corrections of various sorts, however, can often produce a trace anomaly and a breakdown of scale invariance. The (quantum-corrected) black hole solutions of the corresponding gravitational effective field theory (EFT) have a nonvanishing Ricci scalar. Then, the presence of a scalar field with the standard nonminimal coupling $\xi \phi^{2} R$ naturally triggers a spontaneous scalarization of the corresponding black holes. This scalarization phenomenon occurs for an (infinite) discrete set of $\xi$. We illustrate the occurrence of this phenomenon for two examples of static, spherically symmetric, asymptotically flat black hole solution of EFTs. In one example the trace anomaly comes from the matter sector-a novel, closed form generalization of the Reissner-Nordström solution with an $F^{4}$ correction-whereas in the other example it comes from the geometry sector - a noncommutative geometry generalization of the Schwarzschild black hole. For comparison, we also consider the scalarization of a black hole surrounded by (nonconformally invariant) classical matter (Einstein-Maxwell-dilaton black holes). We find that the scalarized solutions are, generically, entropically favored.
\end{abstract}

DOI: 10.1103/PhysRevD.99.084039

\section{INTRODUCTION AND MOTIVATION}

The Schwarzschild black hole $(\mathrm{BH})$ is scale invariant. That is, even though vacuum general relativity introduces a scale, the Planck length, $\ell_{P}$, via Newton's constant, a classical Schwarzschild $\mathrm{BH}$ with a Schwarzschild radius $R_{S}$ of the order of the Planck length $R_{S} \sim \ell_{P}$ and another with $R_{S} \sim$ $10^{9} M_{\odot}$ (like the one at the center of M87 [1]) are identical, up to a scale transformation. This scale invariance remains for all electrovacuum $\mathrm{BH}$ solutions of general relativity (the Kerr-Newman family [2-4]). That is, fixing all other dimensionless parameters, Kerr-Newman BHs with different masses are mapped to one another by a scale transformation. Mathematically, scale invariance is manifest in the tracelessness of the energy-momentum tensor. ${ }^{1}$

This classical symmetry (scale invariance) may become anomalous at the quantum level. For instance, the electromagnetic sector is known to be conformal only classically

\footnotetext{
${ }^{1}$ It is pedagogical to contrast this scale invariance with the scale dependence of other solutions. Consider, for instance, boson stars [5], which are solutions of the Einstein-(complex-) Klein-Gordon model, with a mass term for the scalar field. Boson stars are not scale invariant and have qualitatively different features depending on their total mass. For instance, fixing all other parameters, there can be solutions which are very compact and have a light ring, or less compact solutions without a light ring [6].
}

(and in four spacetime dimensions), by virtue of the running of the coupling induced by the quantum vacuum polarization [7]. At the level of some effective field theory that takes into account the leading quantum effects (e.g., Euler-Heisenberg nonlinear electrodynamics [8]), this anomaly is materialized in the appearance of an energymomentum tensor trace, which generically implies, via the semiclassical Einstein equations, a nonvanishing Ricci scalar. Likewise, most approaches to quantum gravity will introduce a new scale as a cutoff for the validity of the classical geometry, say, the string length in string theory or a noncommutativity parameter in noncommutative geometry (NCG). Generically, this scale will lead to the breakdown of scale invariance in the quantum-corrected $\mathrm{BH}$ solutions and a nonvanishing Ricci scalar.

Apart from a possible quantum origin, classical $\mathrm{BHs}$ with a nonvanishing Ricci scalar are also possible beyond electrovacuum. This occurs when nonconformal matter is present in the action. An example will be presented below. The purpose of this paper is to consider a particular physical effect which can occur for BHs with a nonvanishing Ricci scalar: the phenomenon of spontaneous scalarization of BHs due to a simple and well motivated nonminimal coupling between a scalar field and the Ricci scalar curvature.

Spontaneous scalarization of neutron stars has been discussed for over two decades, since the original proposal [9]. 
It occurs in the context of scalar-tensor theories, wherein a scalar field can be sourced by the trace of the energymomentum tensor. For neutron star geometries, this trace is nonvanishing and, in some regions of the parameter space, it becomes energetically favored for the neutron star to develop a scalar "halo" around it, i.e., to scalarize. Electrovacuum BHs, on the other hand, have a vanishing Ricci scalar and cannot, therefore, source a scalar field and get scalarized in this context $[10,11]$. Still, a similar phenomenon was suggested to also occur for BHs if matter were present in the vicinity of the BH $[12,13]$. Then, Schwarzschild/Kerr BHs could be unstable against such spontaneous scalarization. A concrete realization of this idea was presented in [14] wherein a Kerr BH with synchronized hair [15] was shown to have scalarized counterparts. In these examples, spontaneous scalarization relies on the existence of a nonminimal coupling between the scalar field and the Ricci scalar and may only occur for backgrounds with nonvanishing Ricci scalar, which requires the presence of matter. Moving from the Jordan to the Einstein frame, moreover, the nonminimal coupling to the curvature disappears, and a nonminimal coupling to matter emerges. These observations justify the perspective that this phenomenon is a matter-induced spontaneous scalarization.

On the other hand, a new guise of the spontaneous scalarization phenomenon, dubbed geometric spontaneous scalarization, has recently been under scrutiny. In [16-18] it was pointed out that in gravitational models where a real scalar field minimally couples to the curvature squared Gauss-Bonnet combination, under certain choices of the coupling function, both the standard (bald) vacuum $\mathrm{BH}$ solutions of general relativity and new "hairy" $\mathrm{BH}$ solutions with a scalar field profile are possible, circumventing no-scalar hair theorems [19] (see also [20] for earlier solutions). It was, moreover, suggested that the hairy BHs could form via spontaneous scalarization, since the bald BH solutions were shown to be perturbatively unstable $[16,17]$. Confirming this possibility, however, requires performing dynamical evolutions of the instability in the fully nonlinear theory, which has not been achieved yet. But the suggestion that spontaneous scalarization occurs dynamically for BHs could be confirmed in a cousin model [21]. In this class of models there are no nonminimal couplings between the scalar field and the curvature; there is a nonminimal coupling between the scalar and the electromagnetic field. Thus, it falls in the class of matter-induced spontaneous scalarization, where matter here is the electromagnetic energy. But the chosen source term for the scalar field (the Maxwell invariant) does not require a nonvanishing Ricci scalar for a $\mathrm{BH}$ to scalarize; it requires electromagnetic charge. Scalarization of this sort can occur both for a charged sphere in flat spacetime and for electrically charged BHs; thus, gravity is optional. In this model, fully nonlinear numerical simulations could be performed, showing the unstable bald Reissner-Nordström $(\mathrm{RN}) \mathrm{BHs}$ grow scalar hair, and the growth saturates to match a hairy (or scalarized) solution [21]. Subsequent related work both on geometric and matter-induced spontaneous scalarization of $\mathrm{BHs}$ can be found in [22-30].

A simple, often used in the context of quantum field theory in curved spacetime [31], nonminimal coupling between curvature and a scalar field is of the form $\xi \phi^{2} R$, where $R$ is the Ricci scalar. There are several motivations for this coupling that we shall review below. Since, as argued below, both quantum-corrected $\mathrm{BHs}$ emerging within some effective field theory and classical solutions beyond electrovacuum can have a nonvanishing Ricci scalar, here we study the possibility that spontaneous scalarization exists due to this coupling. Within the quantum considerations we illustrate this possibility with two concrete examples. We shall see that indeed the nonscale invariant $\mathrm{BHs}$ are unstable against scalarization and, moreover, that there are scalarized $\mathrm{BH}$ solutions. The latter are entropically preferred over the scalar-free ones in the model where entropy in unambiguous. These observations support the suggestion that spontaneous scalarization occurs dynamically, even for BHs that would be classically scale invariant, once quantum corrections are taken into account. Considering also an example of a $\mathrm{BH}$ with classically nonscale invariant matter provides evidence that scalarization occurs universally for nonscale invariant BHs, regardless of the classical or quantum origin of the scaleinvariance "anomaly."

This paper is organized as follows. In Sec. II we review the basics of the spontaneous scalarization phenomenon for BHs, discuss the different types thereof and in particular the one that shall be addressed herein, also presenting the specific BH examples that shall be studied in detail. In Sec. III we present the general formalism for obtaining both test field scalar clouds around BHs and the fully nonlinear scalarized solutions. In Secs. IV-VI we present the three illustrative examples of $\mathrm{BH}$ scalarization we have already mentioned. Some conclusions are drawn in Sec. VII.

\section{SCALARIZATION IN A NUTSHELL}

BH scalarization occurs in models described by the Einstein-Hilbert action plus a scalar field action (and plus other possible matter terms). The scalar field action takes the generic form

$$
\mathcal{S}_{\phi}=-\int d^{4} x \sqrt{-g}\left[\frac{1}{2}(\nabla \phi)^{2}+f(\phi) \mathcal{I}(\psi ; g)\right]
$$

where $f(\phi)$ is the coupling function and $\mathcal{I}$ is a source term which generically depends on the metric tensor $g_{\mu \nu}$ and, perhaps, also on some extra matter field(s) $\psi$. For geometric scalarization the latter are not necessary. The corresponding equation of motion for the scalar field $\phi$ reads 


$$
\square \phi=\frac{\partial f}{\partial \phi} \mathcal{I} .
$$

The occurrence of spontaneous scalarization requires two different ingredients. Firstly, there is a scalar-free solution of Eq. (2) with

$$
\phi=\phi_{0}
$$

everywhere. This demands the coupling function should satisfy the condition

$$
\left.\frac{\partial f}{\partial \phi}\right|_{\phi=\phi_{0}}=0
$$

One can set $\phi_{0}=0$ without any loss of generality (via a field redefinition). Thus, the usual vacuum (or electrovacuum) BHs of general relativity also solve the considered model. Secondly, the model should possess another set of solutions with a nontrivial scalar field-the scalarized (or hairy) BHs. These solutions are usually entropically preferred over the scalar-free ones (i.e., they maximize the entropy for given global charges). Moreover, they are smoothly connected with the scalar-free set, approaching it for $\phi=0$.

At the linear level, the spontaneous scalarization phenomenon manifests itself as a tachyonic instability when scalar perturbations of the scalar-free solution are studied. For this analysis one considers a small- $\phi$, denoted $\delta \phi$, expansion of the coupling function

$$
f(\phi)=\left.f\right|_{\phi=0}+\left.\frac{1}{2} \frac{\partial^{2} f}{\partial \phi^{2}}\right|_{\phi=0} \delta \phi^{2}+\mathcal{O}\left(\delta \phi^{3}\right) .
$$

Then, the linearized form of Eq. (2) reads

$$
\left(\square-\mu_{\text {eff }}^{2}\right) \delta \phi=0, \quad \text { where }\left.\mu_{\text {eff }}^{2} \equiv \frac{\partial^{2} f}{\partial \phi^{2}}\right|_{\phi=0} \mathcal{I} .
$$

A tachyonic mass $\mu_{\text {eff }}^{2}<0$ signals an instability of the scalar-free solution; $\mu_{\text {eff }}^{2}<0$ is also the condition for the existence of bound state solutions of Eq. (6). Such bound states mark the onset of the instability. The tachyonic condition can be satisfied for suitable choices of the source $\mathcal{I}$.

The two types of scalarization discussed in the Introduction depend on the "source" term $\mathcal{I}$. Geometric scalarization has been considered using the Gauss-Bonnet invariant $\mathcal{L}_{\mathrm{GB}}$ as the source term in [16-18]

$$
\mathcal{I}=\mathcal{L}_{\mathrm{GB}} .
$$

Similar solutions should exist when taking instead a source term given by the second four-dimensional topological invariant

$$
\mathcal{I}=\mathcal{L}_{C S}
$$

where $\mathcal{L}_{C S}$ is the Pontryagin density, as shown in [26]. The latter, however, requires the presence of rotation for a vacuum $\mathrm{BH}$ to become scalarized.

Matter-induced scalarization is illustrated by the recent work [21], which studied the spontaneous scalarization of electrovacuum BHs, and where the source term was

$$
\mathcal{I}=F_{\mu \nu} F^{\mu \nu}
$$

In this case, the precise form of the coupling function $f(\phi)$ does not seem to be important. The concrete results in [21] were found for $f(\phi)=e^{-\alpha \phi^{2}}$, where the coupling constant $\alpha$ is an input parameter.

\section{A. Scalarization due to the $\phi^{2} R$ nonminimal coupling}

In this work we shall consider matter-induced scalarization with

$$
f(\phi)=\frac{1}{2} \xi \phi^{2} \quad \text { and } \quad \mathcal{I}=R,
$$

i.e., a scalar field Lagrangian

$$
\mathcal{L}_{\phi}=-\frac{1}{2}(\nabla \phi)^{2}-\frac{1}{2} \xi \phi^{2} R,
$$

where $\xi$ is a dimensionless coupling constant, which is an input parameter of the theory.

The nonminimal coupling $\xi \phi^{2} R$ has a long history starting with $[32,33]$ — see also the review discussions in [34-36]. Essentially, a nonminimal coupling $\xi \neq 0$ is sourced by quantum corrections: even if $\xi$ is set to zero in the classical action, renormalization makes $\xi \neq 0$, see also [31]. Thus, $\xi=0$, i.e., "minimal coupling" is a classical value; amongst the nonzero values of $\xi$, "conformal coupling" ( $\xi=1 / 6)$ corresponds to the case for which a massless scalar field theory becomes conformally invariant (in four spacetime dimensions) [31].

The literature on this sort of nonminimal coupling and its physical implications, in particular for cosmology, is vast and we do not intend to review it here. In the context of compact objects, nonetheless, we would like to mention the Bronnikov-Melnikov-Bacharova-Bekenstein (BMBB) BH with conformal scalar hair $[37,38],{ }^{2}$ traversible wormholes $[43,44]$, as well as solitons and BHs with a nonminimally coupled gauged Higgs field [45-47], see also [34,36].

Here, we shall investigate under which circumstances BH scalarization occurs for the simple model (10). From

\footnotetext{
${ }^{2} \mathrm{We}$ remark that the $\mathrm{BMBB} \mathrm{BH}$ is rather special. The spherically symmetric asymptotically flat BHs with generic $\xi$ cannot support nonminimally coupled spatially regular neutral scalar fields [39-42].
} 
the examples studied below, our main conclusion, that we conjecture holds generically, is that given a (static, spherically symmetric, asymptotically flat) $\mathrm{BH}$ with a nonvanishing Ricci scalar, one always finds scalarized generalizations, regular on and outside an event horizon and asymptotically flat, for (infinite) discrete set of $\xi$.

Three concrete BHs with nonvanishing Ricci scalar shall be considered below, of which we shall construct their scalarized counterparts. Firstly, we consider the EinsteinMaxwell-dilaton BH obtained by Gibbons and Maeda [48] and, subsequently, by Garfinkle, Horowitz, and Strominger (GMGHS) [49]. We regard this as an example of nonconformally invariant classical matter, as this model emerges in string theory at tree level. Secondly, we consider an Einstein-Maxwell model with $F^{4}$ correction. This can be faced in the same spirit of the Euler-Heisenberg effective Lagrangian in QED, $\mathcal{L}_{\mathrm{EH}} \propto F^{2}+a\left(F^{2}\right)^{2}+b(F \star F)^{2}$, which accounts for vacuum polarization, but omitting the $\left(F^{2}\right)^{2}$ term. This simplification allows for a simple closed form BH solution which we present here for the first time. We do not expect that including the full EulerHeisenberg Lagrangian, however, should change qualitatively our conclusions concerning scalarization. The final example considers the scalarization of the NCG inspired generalization of the Schwarzschild $\mathrm{BH}$ proposed by Nicolini, Smailagic, and Spallucci (NSS) [50].

In all these examples we start by constructing the corresponding zero modes, or scalar clouds, i.e., infinitesimally small scalar field bound states. For a given $\xi$ these occur for specific background parameters. The continuation at the nonlinear level of these scalar clouds yields the scalarized BHs. For studies of a similar scalarization in the context of horizonless configurations see, e.g., [51,52].

\section{SETUP}

Consider the model described by the action

$$
\mathcal{S}=\int d^{4} x \sqrt{-g}\left[\frac{R}{4}+\mathcal{L}_{0}(\Psi)\right]+\mathcal{S}_{\phi},
$$

where $\mathcal{L}_{0}$ is the Lagrangian for the matter fields, collectively denoted by $\Psi$, and the scalar field action is provided by Eq. (1) with Eq. (10), for the case of interest herein. Variation of action with respect to the metric tensor leads to the Einstein equations

$$
R_{\mu \nu}-\frac{1}{2} R g_{\mu \nu}=2\left(T_{\mu \nu}+T_{\mu \nu}^{\phi}\right),
$$

where $T_{\mu \nu}$ is the energy-momentum tensor associated with $\mathcal{L}_{0}$ and $T_{\mu \nu}^{(\phi)}$ is the one associated with the scalar field action $\mathcal{S}_{\phi}$. The equations of motion for the matter fields can be written once the respective Lagrangian is specified. We assume the existence of a scalar-free $\mathrm{BH}$ solution, with $\phi=0$, which solves the scalar field equation

$$
\nabla^{2} \phi-\xi R \phi=0
$$

$R \neq 0$ allows, in principle, to circumvent the standard Bekenstein-type [53] no-scalar hair theorems. This type of argument is based on constructing an identity that implies triviality of the scalar field. For instance, restricting to static configurations, one constructs from Eq. (14) the identity

$$
\int d^{3} x \sqrt{-g}\left[(\nabla \phi)^{2}+\xi R \phi^{2}\right]=0 .
$$

The kinetic term is everywhere non-negative, but it is clear that if $\xi R<0$ for some space region, relation (14) cannot be used to exclude the existence of solutions.

In the following, we shall be considering the generic spherically symmetric line element

$d s^{2}=-\sigma^{2}(r) N(r) d t^{2}+\frac{d r^{2}}{N(r)}+P^{2}(r)\left(d \theta^{2}+\sin ^{2} \theta d \varphi^{2}\right)$,

where $\sigma, N, P$ are radial functions to be determined. Observe that we have kept some metric gauge freedom, which shall be conveniently fixed later. For future reference, the corresponding Ricci scalar is

$$
\begin{aligned}
R= & -N^{\prime \prime}-N^{\prime}\left(\frac{4 P^{\prime}}{P}+\frac{3 \sigma^{\prime}}{\sigma}\right)-2 N\left(\frac{2 P^{\prime \prime}}{P}+\frac{\sigma^{\prime \prime}}{\sigma}+\frac{2 P^{\prime} \sigma^{\prime}}{P \sigma}\right) \\
& +\frac{2}{P^{2}}\left(1-N P^{\prime 2}\right),
\end{aligned}
$$

where the prime denotes radial derivative.

\section{A. Scalar clouds}

Before studying the fully nonlinear problem it is instructive to consider the limit wherein the scalar field $\phi$ is infinitesimally small, denoted as $\delta \phi$ on the background of the scalar-free $\mathrm{BH}$ solution we assume to exist. Thus, we only have to solve the scalar field equation (14), on the fixed background (16) which solves the Einstein- $\Psi$ equations.

Taking the usual multipolar decomposition

$$
\delta \phi=Y_{\ell m}(\theta, \varphi) U_{\ell}(r),
$$

where $Y_{\ell m}$ are the real spherical harmonics and $\ell, m$ are the associated quantum numbers, i.e., $\ell=0,1, \ldots$ and $-\ell \leq m \leq \ell$, one finds the following radial equation for the function $U_{\ell}$ 


$$
\frac{1}{P^{2} \sigma}\left(P^{2} N \sigma U_{\ell}^{\prime}\right)^{\prime}-\left[\frac{\ell(\ell+1)}{P^{2}}+\xi R\right] U_{\ell}=0
$$

where $R$ is given by Eq. (17).

We are interested in test scalar field configurations around an asymptotically flat, scalar-free $\mathrm{BH}$ background. At the (nonextremal) BH horizon, located at $r=r_{h}$, the metric functions are assumed to have a generic power series expansion of the form

$$
\begin{aligned}
N(r) & =N_{1}\left(r-r_{h}\right)+\cdots, \\
P(r) & =P_{h}+P_{1}\left(r-r_{h}\right)+\cdots, \\
\sigma(r) & =\sigma_{h}+\sigma_{1}\left(r-r_{h}\right)+\cdots,
\end{aligned}
$$

The test scalar field configurations we seek (scalar clouds, or zero modes) are regular at the horizon and vanish asymptotically, being smooth everywhere. At the horizon, the radial function describing the scalar field reads

$$
U_{\ell}(r)=\phi_{0}+\phi_{1}\left(r-r_{h}\right)+\mathcal{O}\left(r-r_{h}\right)^{2}
$$

where, from the scalar field equation,

$$
\phi_{1}=\frac{1}{N_{1} P_{h}^{2}}\left[\ell(\ell+1)+\xi P_{h}^{2} R\left(r_{h}\right)\right] \phi_{0} .
$$

At infinity, on the other hand, asymptotic flatness of the BH background implies

$$
N=1-\frac{2 M}{r}+\cdots, \quad P \rightarrow r, \quad \sigma \rightarrow 1,
$$

where $M$ is the $\mathrm{BH}$ mass. For the scalar field, one finds

$$
U_{\ell}(r)=\frac{Q_{s}}{r^{\ell+1}}+\cdots,
$$

where $Q_{s}$ is a constant. This constant, as well as the scalar field solution interpolating between the horizon and infinity, is found by solving numerically Eq. (19). This corresponds to an eigenvalue problem, which for a given background (and a number $\ell$ ) selects an infinite set of coupling constants $\xi_{n}=\left\{\xi_{0}, \xi_{1}, \ldots\right\}$ labeled by the number $n$ of nodes of the scalar amplitude $U_{\ell}$.

\section{B. The scalarized BHs}

The scalarized BH solutions are the nonlinear continuation of these scalar clouds which solve the full model (12) with nontrivial $\phi$. The scalar field energy-momentum tensor in Eq. (13) is

$$
\begin{aligned}
T_{\mu \nu}^{(\phi)}= & \phi_{, \mu} \phi_{, \nu}-\frac{1}{2} g_{\mu \nu} g^{\alpha \beta} \phi_{, \alpha} \phi_{, \beta} \\
& +\xi\left[\left(R_{\mu \nu}-\frac{1}{2} g_{\mu \nu} R\right) \phi^{2}+g_{\mu \nu} \square \phi^{2}-\phi_{; \mu \nu}^{2}\right] .
\end{aligned}
$$

No other explicit coupling between the scalar field $\phi$ and the other matter fields $\Psi$ will be considered below.

Only the $\ell=0$ (test field) mode leads to a spherically symmetric solution, compatible with the line element (16). Thus, these are the modes we shall focus on here. Moreover, all scalarized BH solutions in this work are found for the metric gauge choice

$$
P(r)=r \quad \text { and } \quad N(r)=1-\frac{2 m(r)}{r}
$$

where $m(r)$ is the Misner-Sharp mass function. With this setup, the equations for the matter fields $\Psi$ are solved together with the Einstein equations for $N(r), \sigma(r)$, and the scalar field, $\phi=U_{0}(r)$. This results in a standard boundary value problem. As mentioned before, the $\mathrm{BH}$ horizon is located at $r=r_{h}>0$ and the expansions (20) and (21) hold to leading order, introducing the positive constants $N_{1}, \sigma_{h}$, $\phi_{0}$. At infinity, the expansions (23) and (24) hold, introducing another two constants: $M$, the Arnowitt-DeserMisner (ADM) mass, and $Q_{s}$, the scalar "charge." The asymptotic behavior of the matter fields $\Psi$ is similar to that in the $\phi=0$ case. Finding a solution of the problem compatible with these asymptotics requires a fine-tuning of the data at the horizon, as specified, e.g., by the value of the scalar field.

For a given value of $\xi$, which is an input parameter of the theory, solving this boundary value problem results in branches of scalarized BHs, which are labeled by an integer $n$, describing the number of nodes of scalar field $\phi$. Only nodeless solutions $(n=0)$ are reported in this work. Furthermore, to simplify the analysis, we shall restrict ourselves herein to the analysis of nonextremal BHs. Finally, we shall not attempt to clarify the critical behavior of solutions.

A particularly relevant quantity in the following is the BH entropy, $S$, of the scalarized BHs. In the absence of a supplementary contribution from $\mathcal{L}_{0}(\Psi)$ in Eq. (12), the expression of $S$, as derived by using Wald's formalism [54,55], possesses an extra contribution with respect to that in Einstein's gravity, due to the nonminimal coupling with the scalar field, and reads

$$
S=\pi r_{h}^{2}\left(1-2 \xi \phi_{0}^{2}\right)
$$

Following standard conventions, we also define the reduced area $a_{H}$, temperature $t_{H}$, entropy $s$, and charge $q$ : 


$$
\begin{array}{rlrl}
a_{H} & \equiv \frac{A_{H}}{16 \pi M^{2}}, & & t_{H} \equiv 8 \pi T_{H} M, \\
s & \equiv \frac{S}{4 \pi M^{2}}, & q \equiv \frac{Q}{M},
\end{array}
$$

where $A_{H}, T_{H}, Q$ are the $\mathrm{BH}$ area, temperature, and electric charge. The latter will only be present for the cases with a Maxwell field below and it will be slightly modified in Sec. V.

\section{SCALARIZED DILATONIC BHS}

\section{A. The scalar-free solution}

Our first example is purely classical. We consider the GMGHS solution $[48,49]$, which provides a simple example of a classical $\mathrm{BH}$ beyond electrovacuum that has a nonvanishing Ricci scalar, being a solution of low energy string theory (for a particular choice of the parameter $a$ introduced below). More concretely, this family of $\mathrm{BH}$ solutions solves Einstein's gravity coupled to a Maxwell field $F=d A$ and a dilaton $\psi$. The corresponding matter action term in Eq. (12) reads

$$
\mathcal{L}_{0}(A, \psi)=-\frac{1}{2}(\nabla \psi)^{2}-\frac{1}{4} e^{-2 a \psi} F^{2},
$$

where $a$ is a free parameter which governs the strength of the coupling between the dilaton and the Maxwell field. ${ }^{3}$ When $a=0$, the action reduces to the usual EinsteinMaxwell theory with a decoupled massless real scalar. When $a=1$, this model is part of the low energy action of string theory; $a=\sqrt{3}$ corresponds to the Kaluza-Klein value, that is, the Einstein-Maxwell-dilaton theory that emerges from the dimensional reduction of vacuum gravity in five spacetime dimensions.

The GMGHS BH solution has the line element (16) with

$$
\begin{aligned}
\sigma(r) & =1, \quad N(r)=\left(1-\frac{r_{+}}{r}\right)\left(1-\frac{r_{-}}{r}\right)^{\frac{1-a^{2}}{1+a^{2}}}, \\
P & =r\left(1-\frac{r_{-}}{r}\right)^{\frac{a^{2}}{1+a^{2}}},
\end{aligned}
$$

together with the Maxwell potential and dilaton field

$$
A=\frac{Q}{r} d t, \quad e^{2 \psi}=\left(1-\frac{r_{-}}{r}\right)^{\frac{2 a}{1+a^{2}}} .
$$

The two free parameters $r_{+}, r_{-}$(with $r_{-}<r_{+}$) are related to the ADM mass, $M$, and (total) electric charge, $Q$, by

\footnotetext{
${ }^{3}$ Changing the sign of $a$ is equivalent to changing the sign of $\psi$; it thus suffices to consider $a \geq 0$.
}

$M=\frac{1}{2}\left[r_{+}+\left(\frac{1-a^{2}}{1+a^{2}}\right) r_{-}\right], \quad Q=\left(\frac{r_{-} r_{+}}{1+a^{2}}\right)^{\frac{1}{2}}$.

For all $a$, the surface $r=r_{+}$is the location of the event horizon. In the extremal limit, which corresponds to the coincidence limit $r_{-}=r_{+}$, the area of the event horizon goes to zero for $a \neq 0$. The Hawking temperature, however, only goes to zero in the extremal limit for $a<1$, while for $a=1$ it approaches a constant, and for $a>1$ it diverges. The Ricci scalar of the GMGHS BHs is strictly positive outside the horizon,

$$
R=\frac{2 a^{2} r_{-}^{2}}{\left(1+a^{2}\right) r^{4}}\left(1-\frac{r_{+}}{r}\right)\left(1-\frac{r_{-}}{r}\right)^{-\frac{1+3 a^{2}}{1+a^{2}}}>0,
$$

which implies that scalarization can occur for negative $\xi$ only. In what follows, we focus on the case $a=1$, although we have verified the occurrence of scalarization for several other values of $a$ as well.

\section{B. The scalarized solutions}

Starting with the analysis of zero modes, we have found that, for any fixed $\ell$, Eq. (19) solved on the GMGHS background with given $M, Q$ admits a family of nontrivial solutions [with $\phi(\infty)=0$ ] for a discrete spectrum of values of the coupling parameter $\xi_{n}$, which are labeled by the node number $n$, although only nodeless solutions $n=0$ are considered here (and $\xi_{0}$ is denoted $\xi$ for simplicity). This feature occurs for any value of the global charges $(M, Q)$.

In Fig. 1 we exhibit the value of $q$ for which the zero mode occurs, as a function of the coupling constant $\xi$. This defines a line- the existence line-which is exhibited for the nodeless zero modes and $\ell=0,1,2$. Recall that this

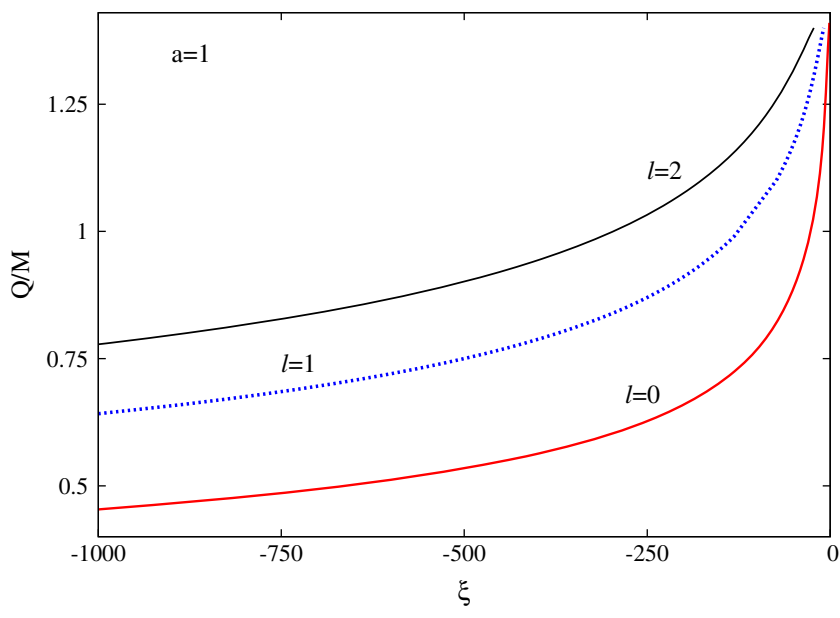

FIG. 1. $\ell=0,1,2$ existence lines for the GMGHS solutions with $a=1$, plotted for $q \equiv Q / M$ in terms of the coupling $\xi$. Scalarization only occurs for negative $\xi$. A larger coupling (in modulus) implies the scalarization instability becomes possible for BHs with smaller $q$. 


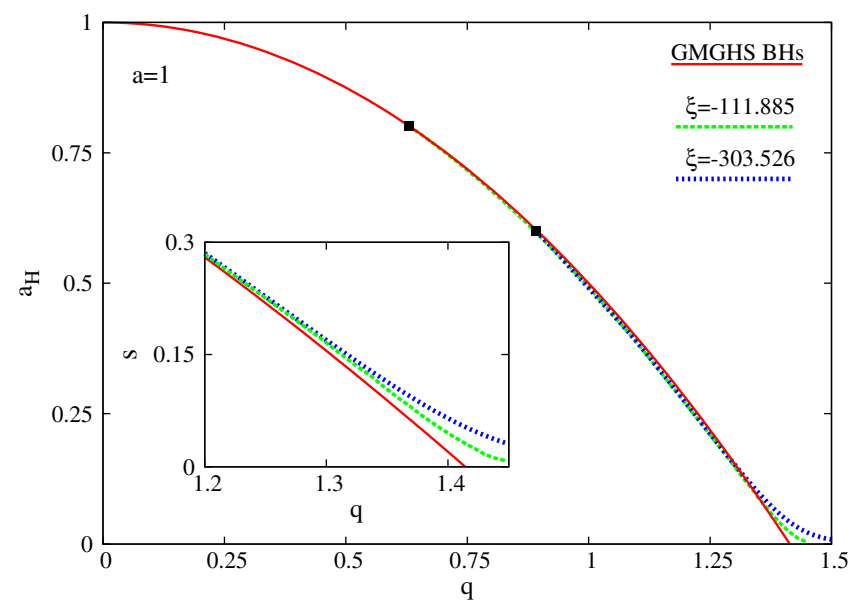

FIG. 2. (Main panel) Reduced area vs $q$ for GMGHS BHs and the corresponding scalarized BHs. The former fall on the solid red line, the latter on the blue and green dashed lines for two values of the coupling. (Inset) Reduced entropy vs $q$ for the same solutions. The shown $q$ interval contains scalarized BHs that have a smaller reduced area than the scalar-free ones, but the reduced entropy is always larger for the former.

zero mode is at the onset of the instability of the scalar-free $\mathrm{BH}$. That is, BHs with a larger (smaller) $q$ than that at which the zero mode occurs (for fixed $\xi, \ell$ ) are unstable (stable) against scalarization due to that $\ell$-mode. Figure 1 shows that the onset of the scalarization instability occurs for BHs with increasingly smaller charge to mass ratio as one increases $|\xi|$. This resembles the pattern observed in [21]. We recall that for the GMGHS solutions $q$ can exceed unity; in fact $q=\sqrt{2 r_{-} / r_{+}}$and thus it has a limiting value of $q=\sqrt{2}$. Only solutions with large $q$ (and greater than unity) can become scalarized for small $|\xi|$, and for each $\ell$ there is a minimum value of $|\xi|$ for which scalarization occurs. The corresponding (approximate) values for $\ell=$ $(0,1,2)$ are, respectively, $(-1.124,-8.877,-22.291)$. In this limit the horizon area goes to zero and $q \rightarrow \sqrt{2}$.

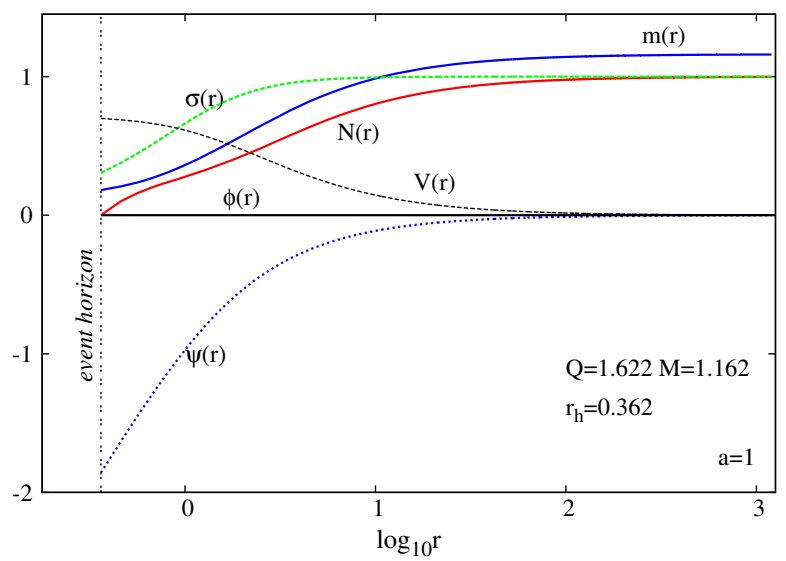

As expected, these scalar zero modes can be continued into the fully nonlinear regime yielding scalarized BHs. For this construction we have only analyzed the spherically symmetric solutions, which bifurcate from the scalar cloud with $\ell=0$, as mentioned before. The bifurcation points, for two different values of $\xi$, together with the corresponding line of scalarized BHs can be seen in Fig. 2, in a reduced area vs $q$ diagram. An interesting feature is that the reduced area of the scalarized BHs can be smaller than that of the comparable scalar-free $\mathrm{BH}$ (with the same q) but the reduced entropy, seen in the inset of the same figure, is always larger, showing the scalarized BHs are entropically favored. The latter conclusion is general for all solutions analyzed.

The profile functions describing the line element, dilaton and scalar fields, and electric potential $V(r)$ of a typical scalarized solution is shown in Fig. 3, comparing it with those for the GMGHS scalar-free solution with the same global charges. The differences are visible although of small magnitude. The variation of the scalar charge for three values of the coupling, in terms of the total mass normalized to the mass of the bifuraction point scalar-free solution and also in terms of the charge to mass ratio, is shown in Fig. 4. Finally, we remark that, similar to the $\phi=0$ case, these solutions seems to possess an extremal limit with vanishing horizon area.

\section{SCALARIZED REISSNER-NORDSTRÖM- $F^{4}$ BHs}

Our second example pertains to a class of quantumcorrected BHs where the quantum correction emerges from the matter sector. It is well known that classical electrodynamics loses conformal invariance due to vacuum polarization. The Euler-Heisenberg effective field theory [8] takes into account the scattering of photons by virtual electron-positron pairs. Integrating the corresponding loop produces an effective quartic interaction vertex between photons, thus a nonlinear theory of electrodynamics which

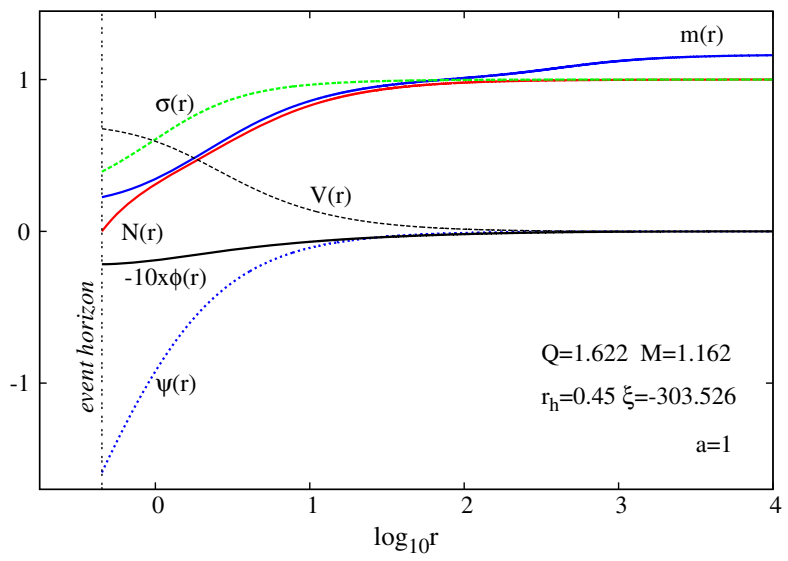

FIG. 3. The profile functions (left panel) of a typical GMGHS solution and (right panel) its scalarized version for the same global charges. 


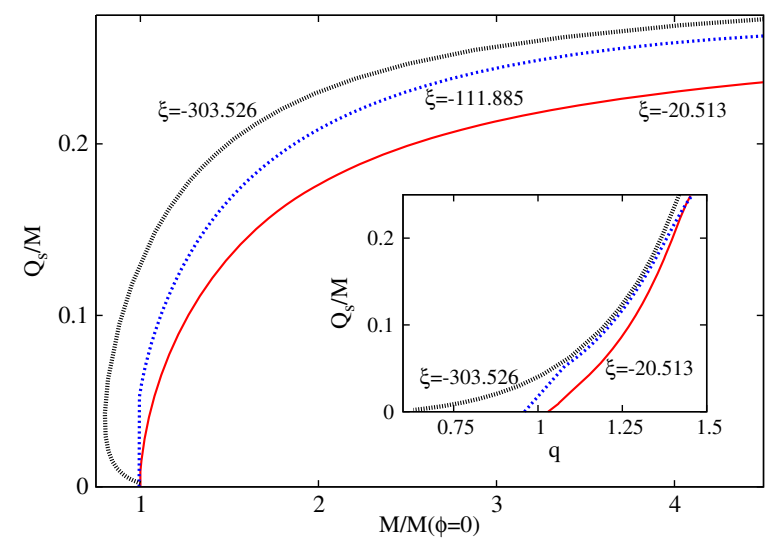

FIG. 4. Variation of the reduced scalar charge with (main panel) the ratio of the ADM mass to the mass of the scalarized solution at the bifurcation point, and (inset) the electric charge to mass ratio, $q$.

is not scale invariant. Another relevant nonlinear theory of electrodynamics emerging from quantum corrections is Born-Infeld theory, in the context of the open string [56]. In the following we shall first remark that, generically, nonlinear electrodynamics breaks down the scale invariance of Maxwell's theory, before presenting a novel BH solution in this context, which we dub $\mathrm{RN}-F^{4} \mathrm{BH}$, which will be analyzed below in the context of scalarization.

\section{A. Scale-invariance breakdown in nonlinear electrodynamics}

Following the discussion in [57], we consider a general Lagrangian $\mathcal{L}=\mathcal{L}(x, y)$ depending on the only two Lorentz and gauge invariant scalars that can be constructed for the electromagnetic field in four spacetime dimensions,

$$
x \equiv \frac{1}{4} F_{\mu \nu} F^{\mu \nu} \quad \text { and } \quad y \equiv \frac{1}{4} F_{\mu \nu}(\star F)^{\mu \nu},
$$

where we have defined the dual field strength as

$$
(\star F)_{\mu \nu}=\frac{1}{2} \epsilon_{\mu \nu \alpha \beta} F^{\alpha \beta},
$$

and where $\epsilon_{\mu \nu \alpha \beta}$ is the Levi-Civita tensor. For such a generic Lagrangian, the corresponding energy-momentum tensor reads

$$
T_{\mu \nu}=\mathcal{L}_{, x} T_{\mu \nu}^{(\text {Maxwell })}+\frac{T}{4} g_{\mu \nu}
$$

where the comma denotes partial derivative, the (usual) Maxwell energy-momentum tensor reads

$$
T_{\mu \nu}^{(\text {Maxwell })}=F_{\mu \alpha} F_{\nu \beta} g^{\alpha \beta}-\frac{1}{4} g_{\mu \nu} F_{\alpha \beta} F^{\alpha \beta},
$$

and the trace is

$$
T \equiv T_{\mu}^{\mu}=-4\left(\mathcal{L}-x \mathcal{L}_{, x}-y \mathcal{L}_{, y}\right) .
$$

One can easily see that the condition $T=0$ is violated in a generic nonlinear Maxwell theory. It follows that the corrected RN BHs emerging in electrovacuum plus nonlinear electrodynamics corrections will, in general, possess a nonvanishing Ricci scalar $R=-T \neq 0$.

\section{B. The scalar-free solution: A new exact BH}

A minimal deviation from standard Maxwell actionwhich corresponds to $\mathcal{L}=x$-requiring both magnetic and electric fields, is found by adding a quadratic term in $y$ and reads $^{4}$

$\mathcal{L}_{0}(A)=x+\alpha y^{2}=\frac{1}{4} F^{2}+\frac{\alpha}{16} F^{4}$, with $F^{4} \equiv\left[F_{\mu \nu}(\star F)^{\mu \nu}\right]^{2}$,

where $\alpha$ is a dimensionful constant, which is a new parameter of the theory. It plays a similar role, say, to the $\alpha$ coupling constant in front of the Gauss-Bonnet (GB) term in the Einstein-GB model. As further motivations to the model (39), we mention that the quartic term occurs, say, in Born-Infeld theory [58] or in the higher loop corrections to the $d=10$ heterotic string low energy effective action. Further motivation can be found in Sec. VII of Ref. [59]. The $\mathrm{BH}$ solutions of a similar model with non-Abelian gauge fields have been studied in [60,61], and possess a variety of interesting solution (e.g., the RN solution possesses nonAbelian hair). We remark that the requirement of a positive energy density imposes $\alpha<0$ in Eq. (39), which is the only case considered in this work.

Model (39) has an energy-momentum tensor with a nonvanishing trace $T=4 \alpha y^{2}$. Thus, the corresponding BH solutions possess a nonzero Ricci scalar and are subject to scalarization in the context of the total action (12). Moreover, we shall now present a new (scalar-free) exact dyonic BH solution which contains some interesting features and that can get scalarized in the context of the present paper.

To find this corrected RN BH, we solve the Einstein equations (13) with an energy-momentum tensor which can be read off from (36). The equations of motion also include the gauge field equations which read

$$
\nabla_{\mu} F^{\mu \nu}+\frac{1}{2} \alpha(\star F)^{\mu \nu}\left[F_{\alpha \beta}(\star F)^{\alpha \beta}\right]_{, \mu}=0 .
$$

\footnotetext{
${ }^{4}$ We recall that $F_{\mu \nu} \star F^{\mu \nu}$ is a total derivative. As such, a linear correction in $y$ to $\mathcal{L}$ leads to the same solutions as in the Maxwell case.
} 
We choose again the metric ansatz (16) and gauge choices (26), and consider a gauge connection which contains both an electric and a magnetic part:

$$
A=V(r) d t+Q_{m} \cos \theta d \varphi,
$$

with $V(r)$ being the electric potential and $Q_{m}$ the magnetic charge. Then, it is straightforward to construct an exact solution of Eqs. (13) and (40). The corresponding expression simplifies by defining the new length scale $r_{0}$

$$
r_{0} \equiv\left(-128 \alpha Q_{m}^{2}\right)^{1 / 4} .
$$

Then, the solutions- the $\mathrm{RN}-F^{4} \mathrm{BHs}$ - are defined by the profile functions

$\sigma(r)=1, \quad N(r)=1-\frac{2 M}{r}+\frac{Q_{m}^{2}+Q^{2}}{r^{2}}+\frac{Q}{r} \Psi(r)$,

$V(r)=\frac{Q}{r}+\Psi(r)$,

and

$$
\begin{aligned}
\Psi(r) \equiv & -\frac{Q}{r}\left\{1+\frac{r}{4 r_{0}}\left[\arctan \left(\frac{\frac{r}{r_{0}}}{1-\frac{r^{2}}{2 r_{0}^{2}}}\right)\right.\right. \\
& \left.\left.+\frac{1}{2} \log \left(\frac{\frac{r^{2}}{2 r_{0}^{2}}-\frac{r}{r_{0}}+1}{\frac{r^{2}}{2 r_{0}^{2}}+\frac{r}{r_{0}}+1}\right)\right]\right\} .
\end{aligned}
$$

As for the standard RN BH, $M, Q$ are constants, corresponding to ADM mass and total electric charge of the solution, respectively.

The position of the (outer) Killing horizon, i.e., the event horizon, is determined as the largest (positive) root of the equation $N\left(r_{h}\right)=0$, as for the standard RN BH. Unlike the latter, however, $r_{h}$ cannot be expressed as a function of $M, Q, Q_{m}$; it is implicitly defined by the equation

$$
\begin{aligned}
M= & \frac{r_{h}}{2}+\frac{Q^{2}+Q_{m}^{2}}{2 r_{h}}-\frac{Q^{2}}{2 r_{h}}\left\{1+\frac{r_{h}}{4 r_{0}}\left[\arctan \left(\frac{\frac{r_{h}}{r_{0}}}{1-\frac{r_{h}^{2}}{2 r_{0}^{2}}}\right)\right.\right. \\
& \left.\left.+\frac{1}{2} \log \left(\frac{\frac{r_{h}^{2}}{2 r_{0}^{2}}-\frac{r_{h}}{r_{0}}+1}{\frac{r_{h}^{2}}{2 r_{0}^{2}}+\frac{r_{h}}{r_{0}}+1}\right)\right]\right\} .
\end{aligned}
$$

The Hawking temperature and area of a spatial section of the event horizon of the solution are

$$
T_{H}=\frac{1}{4 \pi r_{h}}\left[1-\left(\frac{Q_{m}^{2}}{r_{h}^{2}}+\frac{Q^{2} r_{h}^{2}}{4 r_{0}^{4}+r_{h}^{4}}\right)\right], \quad A_{H}=4 \pi r_{h}^{2} .
$$

One can verify that the solutions satisfy the first law of $\mathrm{BH}$ thermodynamics in the form

$$
d M=T_{H} \frac{1}{4} d A_{H}+\Phi_{e} d Q+\Phi_{m} d Q_{m},
$$

with the chemical potentials

$\Phi_{e}=-\frac{Q}{4 r_{0}}\left[\arctan \left(\frac{\frac{r_{h}}{r_{0}}}{1-\frac{r_{h}^{2}}{2 r_{0}^{2}}}\right)+\frac{1}{2} \log \left(\frac{\frac{r_{h}^{2}}{2 r_{0}^{2}}-\frac{r_{h}}{r_{0}}+1}{\frac{r_{h}^{2}}{2 r_{0}^{2}}+\frac{r_{h}}{r_{0}}+1}\right)\right]$,

$\Phi_{m}=\frac{Q_{m}}{r_{h}}$.

The reduced quantities $a_{H}, t_{H}$ can be expressed in a compact form as functions of two parameters $r_{0} / \mathcal{Q}$ and $r_{0} / r_{h}$, where

$$
\mathcal{Q}=\sqrt{Q^{2}+Q_{m}^{2}}
$$

Unlike the $\mathrm{RN}$ case, the corresponding expression are involved and unenlightening and we shall not include them here. They allow, nonetheless, for a fully analytical study of all solutions' properties. The charge to mass ratio is now defined as $q \equiv \mathcal{Q} / M$.

The domain of existence of these solutions with equal electric and magnetic charges is shown in Fig. 5. One observes the existence of overcharged solutions, i.e., with $q>1$, for some range of parameters. Another remark is that the minimal value of the reduced area $a_{H}$ is now $a_{\min }=0.2$, a value attained for $q=q_{\max }=\simeq 1.0278$. The domain of existence is bounded by three curves: (i) the RN limit (i.e., $\alpha \rightarrow 0$ ); (ii) the extremal limit $T_{H} \rightarrow 0$, and (iii) a critical set. This last set is a curious property which is specific to the considered model and can be traced to the presence in Eq. (44) of $\arctan f(r)$ where

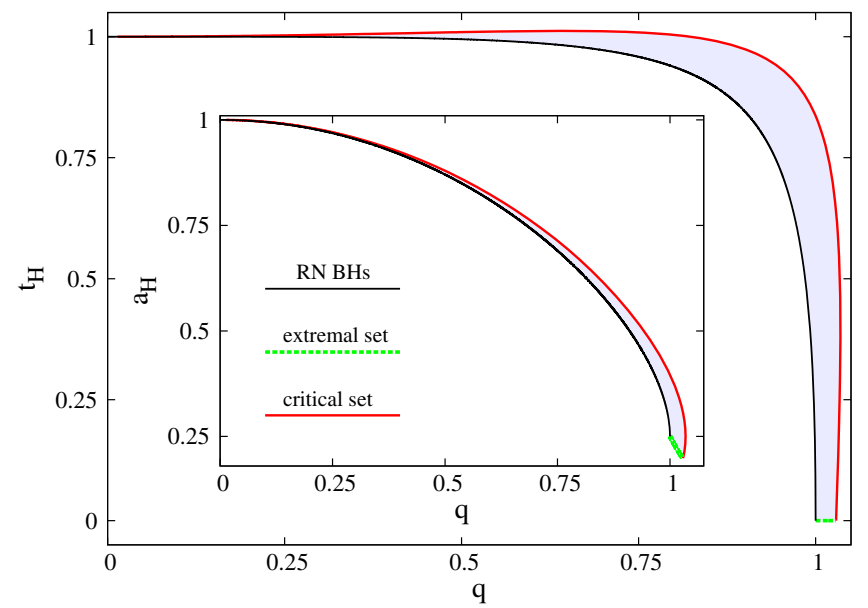

FIG. 5. The domain of existence of RN- $F^{4}$ BHs with $Q=Q_{m}$ in a (main panel) reduced temperature or (inset) reduced area vs charge to mass ratio, $q$, plot. 


$$
f(r)=\frac{\frac{r}{r_{0}}}{1-\frac{r^{2}}{2 r_{0}^{2}}} .
$$

This function possesses a pole at $r_{c}=\sqrt{2} r_{0}$ which implies a different value of the function $\arctan f(r)$ as $r_{c}$ is approached from below or from above. This results in a discontinuity of the metric functions $g_{r r}$ and $g_{t t}$ (as well as of the electric potential) at $r=r_{c}$. Thus, to avoid this pathology to be manifest outside the horizon, we impose

$$
r_{h}>r_{c}=\sqrt{2} r_{0},
$$

which results in the set of critical solutions shown in Fig. 5.

Finally, we remark that the Ricci scalar of the $\mathrm{RN}-F^{4}$ solution is

$$
R=\frac{16 Q^{2} r_{0}^{4}}{\left(r^{4}+4 r_{0}^{4}\right)^{2}}>0
$$

which implies that scalarization can occur for $\xi<0$ only.

\section{The scalarized solutions}

We start, once more, the analysis by considering the zero modes, i.e., the test field scalar clouds that occur at the onset of the scalarization instability on the background of the dyonic $\mathrm{BH}$ described in the previous subsection. No exact solution appears to exist even in this limit and the radial equation (19) is solved numerically. The corresponding results-the existence lines-are shown in Fig. 6, for three different values of $\ell$. Amongst the notable features we highlight (i) the existence of a minimal value of $|\xi|$ below which no zero modes with a given number of nodes exist; (ii) this minimal value of $|\xi|$ increases with $\ell$; and (iii) when varying $\xi$, the zero modes exist for all range of $q=\mathcal{Q} / M$, with $\xi \rightarrow-\infty$ as $q \rightarrow 0$.

The construction of the nonlinear continuation of the scalar clouds follows directly. The far field asymptotics of

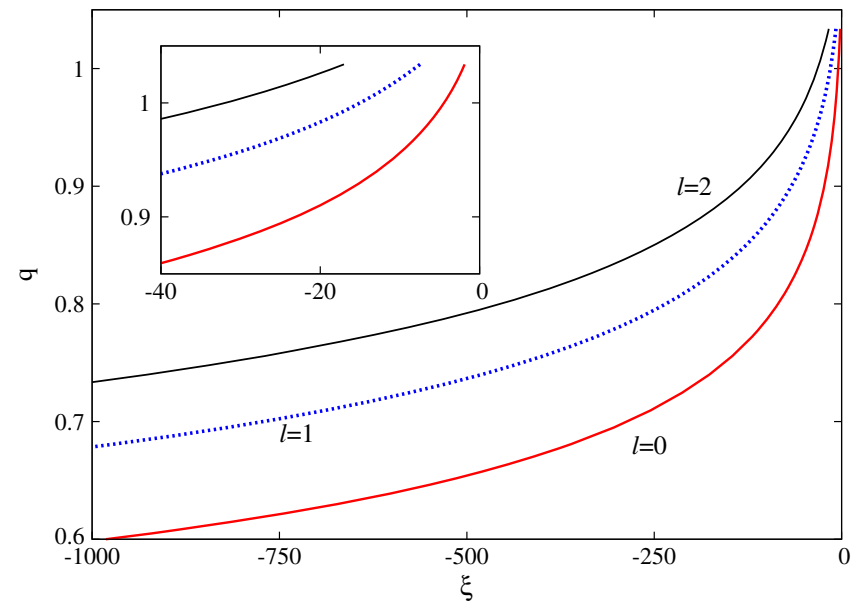

FIG. 6. $\ell=0,1,2$ existence lines for the $\mathrm{RN}-F^{4}$ solution with $Q=Q_{m}=1$. The inset enlarges the region of small $|\xi|$. the scalarized solutions are similar, at leading order, to those of the scalar-free solution (43): $N(r) \rightarrow 1, \sigma(r) \rightarrow 1$, $V(r) \rightarrow \Phi+Q / r$, and $\phi(r) \rightarrow 0$, as $r \rightarrow \infty$. The solution will also possess a horizon at $r=r_{h}>0$, where $N\left(r_{h}\right)=0, V(r)=0$, and $\sigma(r)$ is strictly positive. Then we are left with a system of four nonlinear ordinary differential equations (plus a constraint) for the functions $N, \sigma, \phi$, and $V(r)$. We note that the equation for $V(r)$ possesses the first integral

$$
V^{\prime}=-\frac{Q r^{2} \sigma}{r^{4}+4 r_{0}^{4}},
$$

which allows us to treat $Q$ as an input parameter. Then the scalarized BHs are found by solving numerically two first order equations for $N, \sigma$ and a second order equation for $\phi$. The profile of a typical solution is shown in Fig. 7.

The scalarized solutions still have three global charges $\left(M, Q, Q_{m}\right)$ and, in the region of coexistence, have a larger entropy than the corresponding solutions with $\phi=0$, even though they can have a smaller horizon area-cf. Fig. 8. This figure also shows that one finds, as in the GMGHS case, overcharged solutions with $q>1$. In the right panel of Fig. 8 one observes that, for larger coupling $|\xi|$ the reduced scalar charge grows more slowly in terms of the mass of the solution normalized to that of the bifurcating point. Finally, we notice that the limiting behavior of the branches of scalarized BHs with fixed $\xi$ seems to be similar to that found in the scalar-free case, with the existence of an extremal limit which is regular.

\section{NCG INSPIRED MODIFIED SCHWARZSCHILD BHs}

\section{A. The scalar-free solutions}

As our final example, we consider the NCG inspired Schwarzschild BH, the NSS solution [50]. One may regard this example as taking into account the effects of quantum

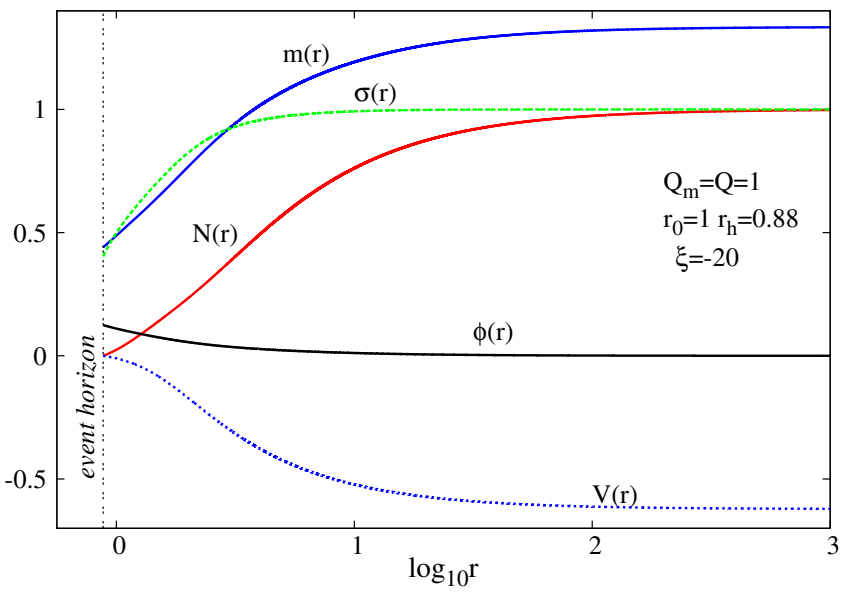

FIG. 7. The profile of a typical scalarized RN- $F^{4}$ solution. 

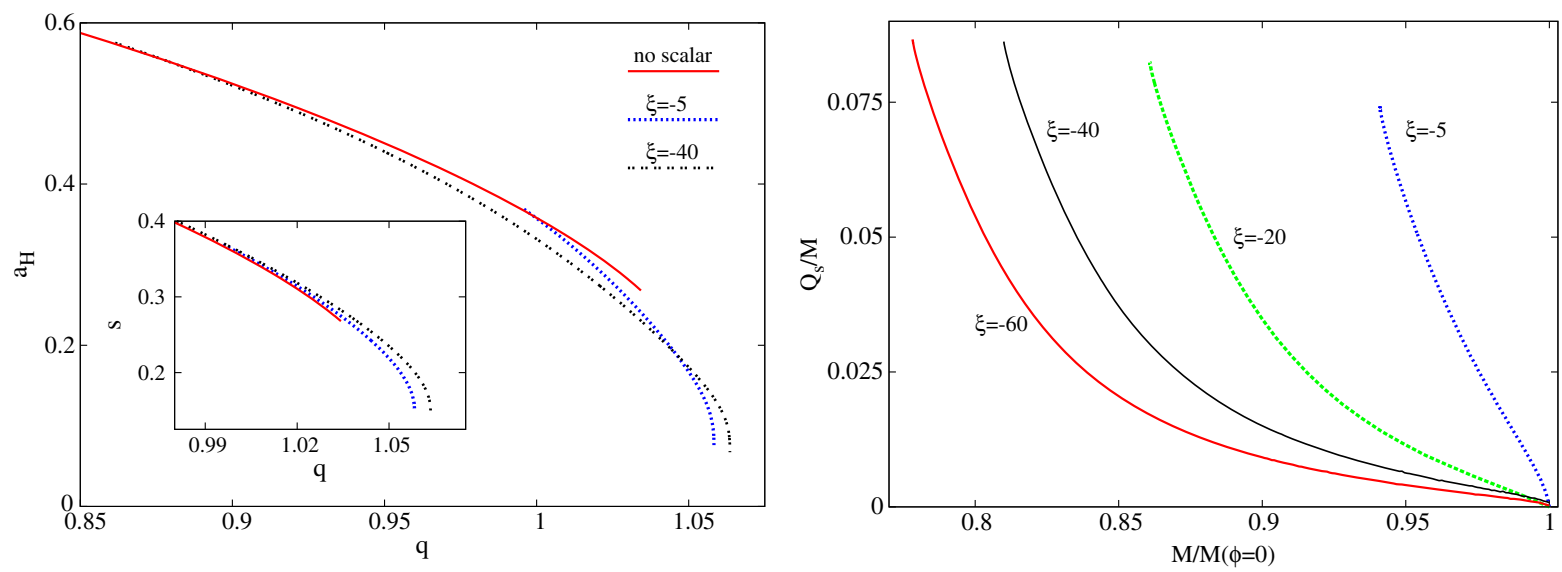

FIG. 8. (Left panel) Reduced area (main panel) and reduced entropy (inset) in terms of the charge to mass ratio $q$ for RN- $F^{4}$ BHs and their scalarized counterparts for two values of the coupling $\xi$. Observe that for fixed $q$ the scalarized solutions are entropically favored. (Right panel) Reduced scalar charge in terms of the ADM mass normalized to the mass at the bifurcation point, for four values of the coupling.

gravity, in the noncommutative geometry approach, yielding a solution which is not scale invariant. The solution solves the Einstein equations (13) with an energy-momentum tensor having the following nonvanishing components:

$$
T_{r}^{r}=p_{r}(r), \quad T_{\theta}^{\theta}=p_{\theta}(r), \quad T_{t}^{t}=-\rho(r),
$$

where

$$
p_{r}(r)=-\rho(r)
$$

Assuming spherical symmetry and taking the metric ansatz (16) with the choices (26), the conservation of this energymomentum tensor, $T_{\mu, \nu}^{\nu}=0$, implies

$$
p_{\theta}(r)=-\rho(r)-\frac{r \rho^{\prime}(r)}{2} .
$$

The NSS solution is obtained postulating a smeared, particlelike source with

$$
\rho(r)=\frac{M}{2 \sqrt{\pi} \vartheta^{3 / 2}} e^{-\frac{r^{2}}{49}},
$$

where $\vartheta$ is an input parameter of the theory related to the spacetime noncommutativity, which introduces a new length scale $r_{0}:^{5}$

$$
\vartheta=\frac{r_{0}^{2}}{4}
$$

Then the Einstein equations (13) yield the following expressions for the metric functions in (16):

\footnotetext{
${ }^{5}$ This choice simplifies a number of relations below. We work in units with $4 \pi G=1$ (while the choice in [50] was $G=1$ ).
}

$$
\sigma(r)=1, \quad N(r)=1-\frac{4 M}{r \sqrt{\pi}} \gamma\left(\frac{3}{2}, \frac{r^{2}}{r_{0}^{2}}\right),
$$

where $\gamma$ is the lower incomplete gamma function

$$
\gamma\left(\frac{3}{2}, \frac{r^{2}}{r_{0}^{2}}\right)=\int_{0}^{r^{2} / r_{0}^{2}} d t t^{1 / 2} e^{-t} .
$$

This solutions possess an (outer) horizon at the $N\left(r_{h}\right)=0$, which fixes the relation between the ADM mass and horizon radius,

$$
M=\frac{\sqrt{\pi} r_{h}}{4 \gamma\left(\frac{3}{2}, \frac{r_{h}^{2}}{r_{0}^{2}}\right)} .
$$

The Hawking temperature and the horizon area of the solutions are

$T_{H}=\frac{1}{4 \pi r_{h}}\left\{1+\frac{2 r_{h}^{2}}{r_{0}^{2}}\left[1-\frac{r_{0}}{2 r_{h}} e^{r_{h}^{2} / r_{0}^{2}} \sqrt{\pi} \operatorname{erf}\left(\frac{r_{h}}{r_{0}}\right)\right]^{-1}\right\}$,

$A_{H}=4 \pi r_{h}^{2}$,

where erf is the error function. We remark that $\mathrm{BHs}$ become cold both in the limit of very large mass (classical limit) and in a new limit where they become extremal $\left(T_{H}=0\right)$, which occurs for $r_{h}=r_{c} \simeq 1.51122 r_{0}$ (i.e., with $\left.A_{H} \neq 0\right)$-see Fig. 4 [50].

Finally, the expression of the Ricci scalar is

$$
R=-\frac{16 e^{-\frac{r^{2}}{r_{0}^{2}}} M\left(r^{2}-2 r_{0}^{2}\right)}{\sqrt{\pi} r_{0}^{5}}<0
$$

which implies that scalarization occurs in this case for $\xi>0$. 


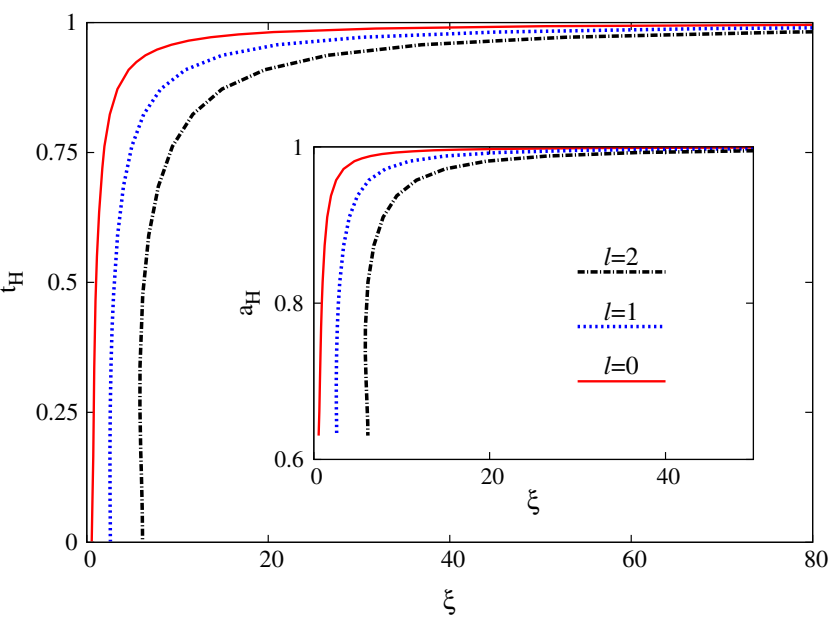

FIG. 9. $\ell=0,1,2$ existence lines for the NSS BHs.

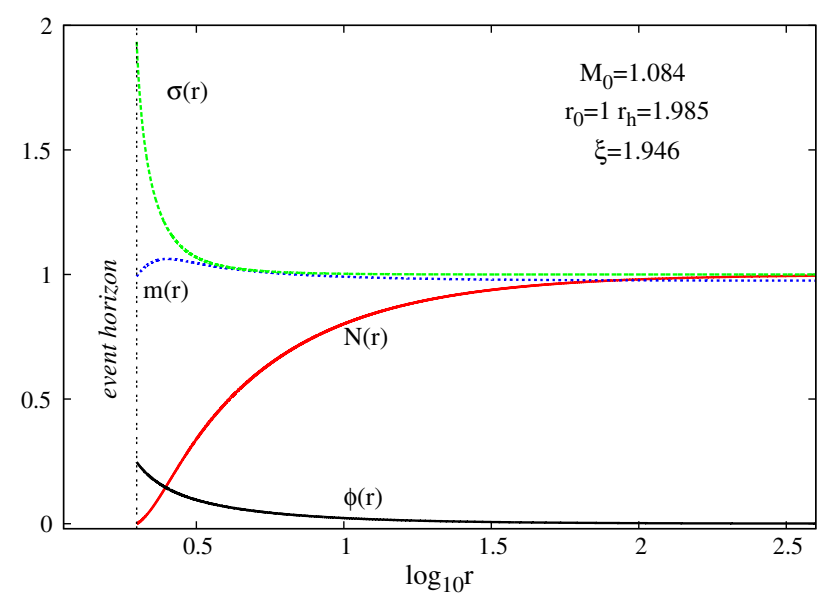

FIG. 10. The profile functions of a typical scalarized NSS solution.

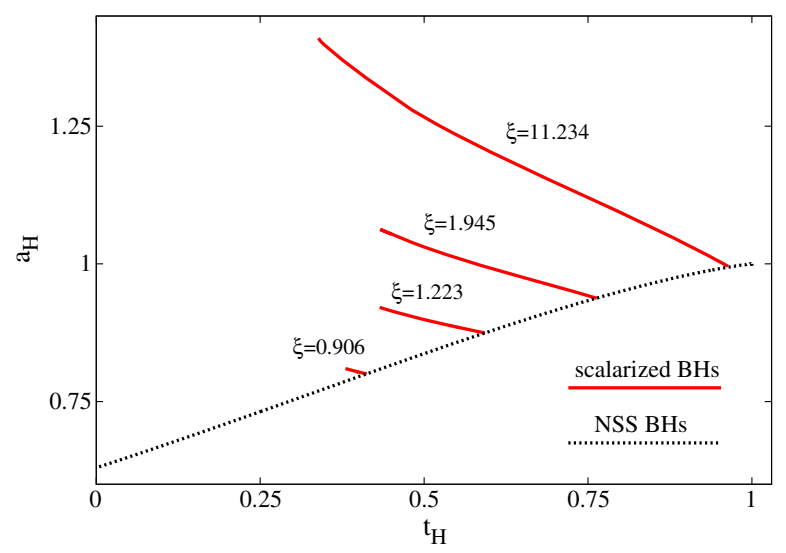

\section{B. The scalarized solutions}

The study of these scalarized solutions is done following closely the approach in the previous two examples. Starting again with the case of an infinitesimally small scalar field, the radial equation (19) is solved for a NSS background. The corresponding existence lines are shown in Fig. 9 for $\ell=0$, 1,2 , in a reduced temperature (or area) vs coupling diagram. Again, scalar clouds exist for any NSS BH, provided one considers a particular set of coupling constants $\xi_{n}$, where $n$ is the node number, although here we always take $n=0$ and denote $\xi_{0} \rightarrow \xi$. One observes that, for a given ADM mass, the value of $\xi$ increases with the Hawking temperature. Approaching the classical (Schwarzschild) limit $\left(r_{0} \rightarrow 0\right)$ scalarization requires $\xi \rightarrow \infty$.

The nonlinear continuation of the scalar zero modes has a subtlety related to the fluid source (54). This source contains a constant fixing the mass of the solutions. In our approach, however, we solve the Einstein equations with an energy-momentum tensor which is the sum of the fluid tensor (54) plus the scalar field contribution (25). For a given model with a specific $\xi$, the constant $M$ in the fluid expression (54) is fixed to be $M=M(\phi=0)$, i.e., the value of the scalar-free solution at the bifurcation point.

A typical scalarized solution is shown in Fig. 10. One can see that, unlike the other two cases discussed above, $m^{\prime}<0$ for large enough values of $r$, which corresponds to occurrence of a region with negative energy density which extends to infinity, although the total mass is still positive for all solutions.

In Fig. 11 (left panel) the bifurcation of the scalarized solutions from the trunk of NSS solutions is shown in a reduced area vs reduced temperature diagram, for several values of the coupling $\xi$. One observes that fixing the temperature the scalarized $\mathrm{BH}$ has always a larger reduced area. For lower temperatures, as the NSS solution is approaching extremality scalarization requires a smaller coupling. One can observe that the extent of the scalarized

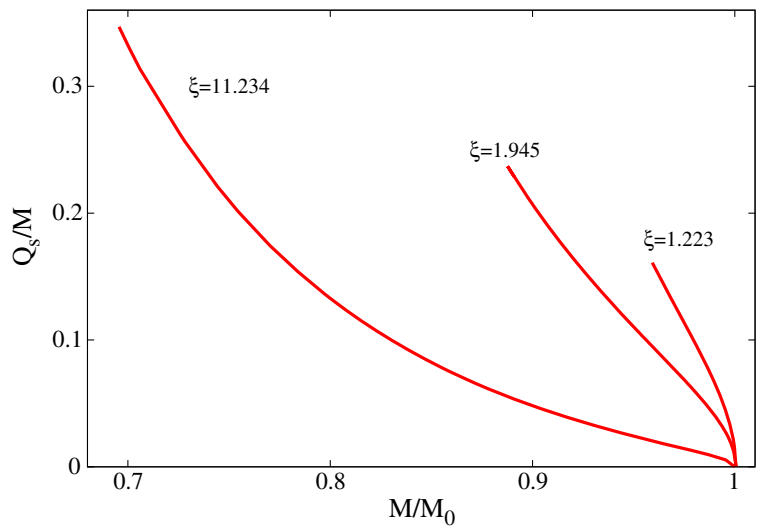

FIG. 11. (Left panel) Branching off of the scalarized solutions from the NSS trunk in a reduced area vs reduced temperature diagram for different values of $\xi$. (Right panel) Reduced scalar charge as a function of the ADM mass of the scalarized NSS BHs normalized to the mass of the bifurcation point. 
branches decreases with $\xi$. In the right panel of Fig. 11 one observes that, again, for smaller couplings the reduced scalar charge increases faster with the mass of the solutions normalized to that of the bifurcation point. Finally, one remarks that, unlike the other two cases above, the answer to the question of which solution maximizes the entropy for given global charges is not unambiguous. Firstly, the consensus in the literature [62] is that the quantum gravity corrections give a correction to the Bekenstein-Hawking formula already in the scalar-free NSS case (which is computed by integrating the first law). A further complication is due to the existence of a "background" fluid source, which provides an extra parameter for the scalarized solutions. Nonetheless, the existence of a spontaneous scalarization instability of the NSS background, which is clear at the linear level, suggests that there should be entropically preferred scalarized solutions.

\section{FURTHER REMARKS}

In this paper we have discussed the possibility that $\mathrm{BH}$ solutions which are not scale invariant, in the sense their energy-momentum tensor is traceful, can scalarize in the presence of a nonminimal coupling between the scalar field and the Ricci scalar curvature. Here, we have considered this scalarization in the context of quantum field theory motivated nonminimal coupling $\xi \phi^{2} R$, but similar conclusions can be extracted for more general scalar-tensor theories.

We have considered three illustrative examples of nonscale invariant BHs. In two of the considered cases the breakdown of scale invariance can be attributed to quantum effects (a sort of trace anomaly) whereas in the remaining case it is due to the presence of scale-invariance braking classical matter. In all cases the pattern is similar. The scalar-free solutions become prone to a tachyonic instability in some region of the parameter space. In particular, at the onset of the instability there is a zero mode (scalar cloud) that we have computed in all examples. Then scalarized solutions branch off from the scalar-free ones at bifurcating points corresponding to the latter solutions that can support the scalar clouds. Since the scalarized solutions are entropically preferred (in the cases the entropy is unambiguous) for the same global charges it seems reasonable to anticipate that they will be the end point of the instability observed for the scalar-free solutions. This was dynamically confirmed in [21] for another model of matter-induced spontaneous scalarization. Establishing a similar result in the present cases also requires fully nonlinear numerical simulations.

Let us conclude with some possible further avenues of related research. Firstly, other features of the scalarized BHs unveiled in [21] should occur also in this case. For example, we predict the existence of static BHs without isometries also for this type of scalarization, branching off from the scalarfree trunk at zero modes with higher $\ell$ and azimuthal quantum number $m \neq 0$. Secondly, it would be interesting to further investigate the scalarized BHs for other, potentially more realistic generalizations of Schwarzschild BHs, within quantum gravity frameworks. Thirdly, one could consider higher dimensions and different asymptotics. In particular, concerning (anti-)de Sitter asymptotics, BHs with scalar hair are known to exist, being supported by the $\xi \phi^{2} R$ term $[63,64]$. It would be interesting to reconsider them in the context of spontaneous scalarization. Finally, it would be interesting to investigate the connection of the scalarization mechanism discussed in this work with the quantum instabilities due to a nonminimally coupling ("awaking the vacuum”) discussed, e.g., in [65-67].

\section{ACKNOWLEDGMENTS}

E. R. thanks D. H. Tchrakian for valuable discussions and for collaboration on related projects. This work has been supported by the FCT (Portugal) IF program, by FCT Grant No. PTDC/FIS-OUT/28407/2017, by Centro de Investigação e Desenvolvimento em Matemática e Aplicações (FCT) Strategic Project No. UID/MAT/04106/2013, by Centro Multidisciplinar de Astrofísica (FCT) Strategic Project No. UID/FIS/00099/2013 and by the European Union's Horizon 2020 Research and Innovation (RISE) programs H2020-MSCA-RISE-2015 Grants No. StronGrHEP-690904 and H2020-MSCA-RISE-2017 Grant No. FunFiCO-777740. The authors would like to acknowledge networking support by the COST Action CA16104. E. R. is grateful for hospitality and support to Dublin Institute for Advanced Studies, where a part of this work was done.
[1] F. Macchetto, A. Marconi, D. J. Axon, A. Capetti, W. Sparks, and P. Crane, The supermassive black hole of M87 and the kinematics of its associated gaseous disk, Astrophys. J. 489, 579 (1997).

[2] R. P. Kerr, Gravitational Field of a Spinning Mass as an Example of Algebraically Special Metrics, Phys. Rev. Lett. 11, 237 (1963).
[3] E. T. Newman, R. Couch, K. Chinnapared, A. Exton, A. Prakash, and R. Torrence, Metric of a rotating, charged mass, J. Math. Phys. (N.Y.) 6, 918 (1965).

[4] P. T. Chruściel, J. Lopes Costa, and M. Heusler, Stationary black holes: Uniqueness and beyond, Living Rev. Relativity 15, 7 (2012). 
[5] F. E. Schunck and E. W. Mielke, General relativistic boson stars, Classical Quantum Gravity 20, R301 (2003).

[6] P. V. P. Cunha, C. A. R. Herdeiro, E. Radu, and H. F. Runarsson, Shadows of Kerr Black Holes with Scalar Hair, Phys. Rev. Lett. 115, 211102 (2015).

[7] M. E. Peskin and D. V. Schroeder, An Introduction to Quantum Field Theory (Addison-Wesley, Reading, MA, 1995).

[8] W. Heisenberg and H. Euler, Conclusions from Dirac's theory of the positron, Z. Phys. 98, 714 (1936).

[9] T. Damour and G. Esposito-Farese, Nonperturbative Strong Field Effects in Tensor-Scalar Theories of Gravitation, Phys. Rev. Lett. 70, 2220 (1993).

[10] S. W. Hawking, Black holes in the Brans-Dicke theory of gravitation, Commun. Math. Phys. 25, 167 (1972).

[11] T. P. Sotiriou and V. Faraoni, Black Holes in Scalar-Tensor Gravity, Phys. Rev. Lett. 108, 081103 (2012).

[12] V. Cardoso, I. P. Carucci, P. Pani, and T. P. Sotiriou, Black Holes with Surrounding Matter in Scalar-Tensor Theories, Phys. Rev. Lett. 111, 111101 (2013).

[13] V. Cardoso, I. P. Carucci, P. Pani, and T. P. Sotiriou, Matter around Kerr black holes in scalar-tensor theories: Scalarization and superradiant instability, Phys. Rev. D 88, 044056 (2013).

[14] B. Kleihaus, J. Kunz, and S. Yazadjiev, Scalarized hairy black holes, Phys. Lett. B 744, 406 (2015).

[15] C. A. R. Herdeiro and E. Radu, Kerr Black Holes with Scalar Hair, Phys. Rev. Lett. 112, 221101 (2014).

[16] D. D. Doneva and S. S. Yazadjiev, New Gauss-Bonnet Black Holes with Curvature Induced Scalarization in the Extended Scalar-Tensor Theories, Phys. Rev. Lett. 120, 131103 (2018).

[17] H. O. Silva, J. Sakstein, L. Gualtieri, T. P. Sotiriou, and E. Berti, Spontaneous Scalarization of Black Holes and Compact Stars from a Gauss-Bonnet Coupling, Phys. Rev. Lett. 120, 131104 (2018).

[18] G. Antoniou, A. Bakopoulos, and P. Kanti, Evasion of NoHair Theorems and Novel Black-Hole Solutions in GaussBonnet Theories, Phys. Rev. Lett. 120, 131102 (2018).

[19] C. A. R. Herdeiro and E. Radu, Asymptotically flat black holes with scalar hair: A review, Int. J. Mod. Phys. D 24, 1542014 (2015).

[20] P. Kanti, N. E. Mavromatos, J. Rizos, K. Tamvakis, and E. Winstanley, Dilatonic black holes in higher curvature string gravity, Phys. Rev. D 54, 5049 (1996).

[21] C. A. R. Herdeiro, E. Radu, N. Sanchis-Gual, and J. A. Font, Spontaneous Scalarization of Charged Black Holes, Phys. Rev. Lett. 121, 101102 (2018).

[22] G. Antoniou, A. Bakopoulos, and P. Kanti, Black-hole solutions with scalar hair in Einstein-scalar-Gauss-Bonnet theories, Phys. Rev. D 97, 084037 (2018).

[23] J. L. Blázquez-Salcedo, D. D. Doneva, J. Kunz, and S. S. Yazadjiev, Radial perturbations of the scalarized EinsteinGauss-Bonnet black holes, Phys. Rev. D 98, 084011 (2018).

[24] Y.S. Myung and D.-C. Zou, Instability of ReissnerNordström black hole in Einstein-Maxwell-scalar theory, arXiv:1808.02609.

[25] D. D. Doneva, S. Kiorpelidi, P. G. Nedkova, E. Papantonopoulos, and S.S. Yazadjiev, Charged GaussBonnet black holes with curvature induced scalarization in the extended scalar-tensor theories, Phys. Rev. D 98, 104056 (2018).

[26] Y. Brihaye, C. Herdeiro, and E. Radu, The scalarised Schwarzschild-NUT spacetime, Phys. Lett. B 788, 295 (2019).

[27] M. Boskovic, R. Brito, V. Cardoso, T. Ikeda, and H. Witek, Axionic instabilities and new black hole solutions, Phys. Rev. D 99, 035006 (2019).

[28] M. Minamitsuji and T. Ikeda, Scalarized black holes in the presence of the coupling to Gauss-Bonnet gravity, Phys. Rev. D 99, 044017 (2019).

[29] Y. S. Myung and D.-C. Zou, Quasinormal modes of scalarized black holes in the Einstein-Maxwell-scalar theory, Phys. Lett. B 790, 400 (2019).

[30] H. O. Silva, C. F. B. Macedo, T. P. Sotiriou, L. Gualtieri, J. Sakstein, and E. Berti, Stability of scalarized black hole solutions in scalar-Gauss-Bonnet gravity, Phys. Rev. D 99, 064011 (2019).

[31] N. D. Birrell and P. C. W. Davies, Quantum Fields in Curved Space (Cambridge University Press, Cambridge, England, 1982).

[32] N. A. Chernikov and E. A. Tagirov, Quantum theory of scalar fields in de Sitter space-time, Ann. Inst. Henri Poincaré, A 9, 109 (1968).

[33] C. G. Callan, Jr., S. R. Coleman, and R. Jackiw, A new improved energy-momentum tensor, Ann. Phys. (N.Y.) 59, 42 (1970).

[34] S. Capozziello and M. De Laurentis, Extended theories of gravity, Phys. Rep. 509, 167 (2011).

[35] V. Faraoni, A crucial ingredient of inflation, Int. J. Theor. Phys. 40, 2259 (2001).

[36] V. Faraoni, E. Gunzig, and P. Nardone, Conformal transformations in classical gravitational theories and in cosmology, Fundam. Cosm. Phys. 20, 121 (1999).

[37] J. D. Bekenstein, Exact solutions of Einstein conformal scalar equations, Ann. Phys. (N.Y.) 82, 535 (1974).

[38] N. M. Bocharova, K. A. Bronnikov, and V. N. Melnikov, An exact solution of the system of Einstein equations and massfree scalar field, Vestn. Mosk. Univ., Ser. 3: Fiz., Astron. 6, 706 (1970).

[39] A. E. Mayo and J. D. Bekenstein, No hair for spherical black holes: Charged and nonminimally coupled scalar field with self-interaction, Phys. Rev. D 54, 5059 (1996).

[40] J. D. Bekenstein, Black hole hair: 25 years after, in Proceedings of 2nd International A.D. Sakharov Conference on Physics, Moscow, 1996, edited by I. M. Dremin and A. M. Semikhatov (World Scientific, Singapore, 1997), p. 761.

[41] S. Hod, No hair for spherically symmetric neutral black holes: Nonminimally coupled massive scalar fields, Phys. Rev. D 96, 124037 (2017).

[42] S. Hod, No nonminimally coupled massless scalar hair for spherically symmetric neutral black holes, Phys. Lett. B 771, 521 (2017).

[43] C. Barcelo and M. Visser, Traversable wormholes from massless conformally coupled scalar fields, Phys. Lett. B 466, 127 (1999).

[44] C. Barcelo and M. Visser, Scalar fields, energy conditions, and traversable wormholes, Classical Quantum Gravity 17, 3843 (2000). 
[45] J. J. van der Bij and E. Radu, Regular and black hole solutions of the Einstein-Yang-Mills-Higgs equations: The case of nonminimal coupling, Nucl. Phys. B585, 637 (2000).

[46] A. V. Nguyen and K. C. Wali, Magnetic monopoles in the Einstein-Yang-Mills-Higgs system, Phys. Rev. D 51, 1664 (1995).

[47] Y. Brihaye and Y. Verbin, Self-gravitating spherical solutions of the nonminimally coupled non-Abelian Higgs model, Phys. Rev. D 91, 064021 (2015).

[48] G. W. Gibbons and K.i. Maeda, Black holes and membranes in higher dimensional theories with dilaton fields, Nucl. Phys. B298, 741 (1988).

[49] D. Garfinkle, G. T. Horowitz, and A. Strominger, Charged black holes in string theory, Phys. Rev. D 43, 3140 (1991); Erratum, Phys. Rev. D 45, 3888(E) (1992).

[50] P. Nicolini, A. Smailagic, and E. Spallucci, Noncommutative geometry inspired Schwarzschild black hole, Phys. Lett. B 632, 547 (2006).

[51] M. Salgado, D. Sudarsky, and U. Nucamendi, On spontaneous scalarization, Phys. Rev. D 58, 124003 (1998).

[52] A. Fuzfa, M. Rinaldi, and S. Schlogel, Particlelike Distributions of the Higgs Field Nonminimally Coupled to Gravity, Phys. Rev. Lett. 111, 121103 (2013).

[53] J. D. Bekenstein, Transcendence of the Law of BaryonNumber Conservation in Black Hole Physics, Phys. Rev. Lett. 28, 452 (1972).

[54] R. M. Wald, Black hole entropy is the Noether charge, Phys. Rev. D 48, R3427 (1993).

[55] V. Iyer and R. M. Wald, Some properties of Noether charge and a proposal for dynamical black hole entropy, Phys. Rev. D 50, 846 (1994).
[56] E. S. Fradkin and A. A. Tseytlin, Nonlinear electrodynamics from quantized strings, Phys. Lett. 163B, 123 (1985).

[57] G. W. Gibbons and C. A. R. Herdeiro, Born-Infeld theory and stringy causality, Phys. Rev. D 63, 064006 (2001).

[58] A. A. Tseytlin, On non-Abelian generalization of BornInfeld action in string theory, Nucl. Phys. B501, 41 (1997).

[59] A. Maleknejad and M. M. Sheikh-Jabbari, Non-Abelian gauge field inflation, Phys. Rev. D 84, 043515 (2011).

[60] E. Radu and D. H. Tchrakian, Stable black hole solutions with non-Abelian fields, Phys. Rev. D 85, 084022 (2012).

[61] C. Herdeiro, V. Paturyan, E. Radu, and D. H. Tchrakian, Reissner-Nordström black holes with non-Abelian hair, Phys. Lett. B 772, 63 (2017).

[62] P. Nicolini, Noncommutative black holes, the final appeal to quantum gravity: A review, Int. J. Mod. Phys. A 24, 1229 (2009).

[63] E. Winstanley, On the existence of conformally coupled scalar field hair for black holes in (anti-)de Sitter space, Found. Phys. 33, 111 (2003).

[64] E. Radu and E. Winstanley, Conformally coupled scalar solitons and black holes with negative cosmological constant, Phys. Rev. D 72, 024017 (2005).

[65] R. F. P. Mendes, G. E. A. Matsas, and D. A. T. Vanzella, Quantum versus classical instability of scalar fields in curved backgrounds, Phys. Rev. D 89, 047503 (2014).

[66] W. C. C. Lima, G. E. A. Matsas, and D. A. T. Vanzella, Awaking the Vacuum in Relativistic Stars, Phys. Rev. Lett. 105, 151102 (2010).

[67] A. G. S. Landulfo, W. C. C. Lima, G. E. A. Matsas, and D. A. T. Vanzella, Particle creation due to tachyonic instability in relativistic stars, Phys. Rev. D 86, 104025 (2012). 